\title{
A survey on knowledge and attitudes towards molar-Incisor hypomineralization among undergraduate and postgraduate students in School of Stomatology, Wuhan University
}

\section{Yanchen Liu}

The State Key Laboratory Breeding Base of Basic Science of Stomatology (Hubei-MOST) \& Key Laboratory of Oral Biomedicine Ministry of Education, School and Hospital of Stomatology, Wuhan University

Miao He ( $\sim$ hemiao@whu.edu.cn )

The State Key Laboratory Breeding Base of Basic Science of Stomatology (Hubei-MOST) \& Key Laboratory of Oral Biomedicine Ministry of Education, School and Hospital of Stomatology, Wuhan University

\section{Research Article}

Keywords: Clinical management, Dental developmental disease, Molar-incisor hypomineralization

Posted Date: October 20th, 2021

DOI: https://doi.org/10.21203/rs.3.rs-957678/v1

License: (c) (i) This work is licensed under a Creative Commons Attribution 4.0 International License. Read Full License 


\section{Abstract}

Background: Molar incisor hypomineralization $(\mathrm{MIH})$ is a developmental dental disease, and its clinical management challenges dentists. The study aimed to investigate the knowledge about $\mathrm{MIH}$ and attitudes towards learning more about MIH among undergraduate and postgraduate students in the School of Stomatology, Wuhan University.

Methods: This survey was based on a questionnaire, modified based on previous studies. The questionnaire was sent to 540 undergraduate and postgraduate students from the School of Stomatology, Wuhan University. Questions covered clinical experience, perceptions, clinical management, and preferences for further training. Data were analyzed with the Chi-square test.

Results: We collected 368 questionnaires (response rate: 68\%). Among those, 89\% (328/368) were eligible for analysis. Most respondents ( $80 \%$ ) had heard of $\mathrm{MIH}$, primarily from classroom teaching. However, only $40 \%$ of the students had observed the disease clinically, and a relatively low proportion of students were familiar with the etiology, incidence, differential diagnosis, and treatment of MIH. Most respondents were highly enthusiastic and had high expectations about further systematic teaching about $\mathrm{MIH}$.

Conclusion: Most students in this study had heard of $\mathrm{MIH}$, but few were familiar with the principles of diagnosis and differential diagnosis. Systematic teaching about MIH is warranted.

\section{Background}

Molar-incisor mineralization $(\mathrm{MIH})$ is a disease that causes the hypomineralization of tooth enamel. It was first proposed by Weerjihim in $2001^{1}$, and it was officially defined as an independent disease by the European Society of Pediatric Dentistry in $2003^{2}$. The etiology of MIH remains unknown. It is currently believed to be related to genetic factors, environmental factors, drug factors, and general conditions that occur during pregnancy and in the first three years after birth ${ }^{3}$. The global average incidence of $\mathrm{MIH}$ is $14.2 \%{ }^{4}$, but it varies greatly from country to country. The incidence of $\mathrm{MIH}$ in children 6-12 years old in China ranges from 4.45 to $25.5 \% 5,6$. The clinical manifestations of teeth mildly affected by MIH include clearly defined white and light-yellow plaques. In severe cases, the enamel may breakdown after eruption, and cannot be repaired; in these cases, the teeth are typically extracted early ${ }^{2}$. There is no unified opinion on a treatment for $\mathrm{MIH}$, but currently, treatments mainly aim to relieve symptoms. Previous studies have found that the oral health-related quality of life of children with $\mathrm{MIH}$ was lower than that of children without $\mathrm{MIH}^{7}$. The main reasons for low quality of life included tooth sensitivity, which caused chewing discomfort, and colored plaques on the front teeth, which caused aesthetic problems ${ }^{8}$.

For dentists, it is important to distinguish $\mathrm{MIH}$ from other dental developmental defects. MIH has gradually attracted attention over the years, and several surveys have been conducted on dentist awareness of $\mathrm{MIH}^{9-\mathrm{c} 11}$. Dentists believe that $\mathrm{MIH}$ management presents quite a challenge. To date, only two surveys on student awareness have been conducted; one in Germany and one in Saudi Arabia ${ }^{12,13}$. 
Given the high prevalence of MIH in China and the lack of awareness among dental students, the present study aimed to investigate the knowledge of $\mathrm{MIH}$ and attitudes towards learning more about $\mathrm{MIH}$ among undergraduate and postgraduate students in the School of Stomatology, Wuhan University. We also evaluated whether knowledge of MIH was different among students of different academic levels. The results of this study might influence future curriculum planning.

\section{Methods}

\section{Study design and procedures}

This survey included undergraduates (fourth- and fifth-year undergraduates) and postgraduates (first-, second-, and third-year postgraduates) enrolled in the School of Stomatology, Wuhan University, in January 2021. All current students were included in the sample. Prior to participant enrollment, this study was approved by the Medical Ethics Committee of the School of Stomatology, Wuhan University (\#HGGC076). Before the questionnaire was delivered, we conducted a pre-survey on the questionnaire to ensure that the questions were readily understood. For this study, the questionnaire was provided in electronic form and distributed in public discussion groups (WeChat and QQ software platforms, Tencent Corporate, Shenzhen, China), with assistance from the Wenjuanxing (Questionnaire Collection Software, Ranxing Technology Corporate, Changsha, China). All questionnaires were completed voluntarily and anonymously. One week after the questionnaire was distributed, all the participating groups were notified again, as a reminder.

\section{Survey instrument}

The questionnaire was composed of three parts. The first part contained basic information, including each participant's academic level. The second part included disease awareness; the source of knowledge acquisition; the degree of mastery over MIH etiology, epidemiology, clinical manifestations, and differential diagnosis; the probability of clinical encounters; and the choice of treatment methods. The third part included the following three questions: Do you want to gain relevant knowledge about $\mathrm{MIH}$ ? What would you most like to know? What are the principal ways to obtain professional dental knowledge? Between the first and second parts of the questionnaire, a brief introduction to $\mathrm{MIH}$ was provided to clarify the subject of the questionnaire and avoid errors in understanding. The related pictures of MIH used in questionnaire was the treatment manual, published by Ghanim et al. (2017) ${ }^{14}$.

To avoid blank items in the questionnaire, the questionnaire was set to skip non-applicable questions automatically. For example, when a respondent had not been involved in treating $\mathrm{MIH}$, they were not asked about the preferred materials commonly used in clinical practice for treating MIH.

\section{Statistical analyses}

We performed all statistical analyses with SPSS Ver. 26.0 (IBM, NY, USA). The descriptive statistical analyses are presented in distribution tables and frequency tables. The distributions were compared with 
the chi-square test. The reliability and validity of the questionnaire were verified with the Cronbach a index and Kaiser-Meyer-Olkin (KMO) value, respectively. The threshold for a statistical difference was set to 0.01 .

\section{Results}

We collected 368 questionnaires from students in five different academic levels. The response rate of the questionnaire was $68 \%(368 / 540)$ and the effective rate of the questionnaire was $89 \%$ (328/368; Table 1). The reliability of the questionnaire was 0.852 (Cronbach's a index) and the validity of the questionnaire was 0.889 (KMO test).

Most students $(264 / 328,80 \%)$ had heard of $\mathrm{MIH}$, and we found no significant difference between undergraduate and postgraduate students $\left(\lambda^{2}=5.513, p>0.01\right.$; Table 3$)$. Moreover, most students had heard about MIH in more than one way. The most common source was classroom teaching $(188 / 264$, $71 \%)$, followed by clinical practice $(111 / 264,42 \%)$, lectures $(56 / 264,21 \%)$, journal articles $(37 / 264,14 \%)$, and other sources (15/264,6\%; Table 3). Some students (104/264, 39\%) had observed MIH clinically (Table 2). The proportions of students that had observed MIH clinically was significantly different among the different academic levels in $\left(\lambda^{2}=62.928, p<0.01\right.$; Table 2$)$. However, only 66 students $(66 / 264,25 \%)$ were confident in identifying $\mathrm{MIH}$ (Table 2), and the ability to identify MIH was significantly different among the different academic levels $\left(\lambda^{2}=65.267, p<0.01\right.$; Table 2$)$. Few students $(43 / 264,16 \%)$ knew the diagnostic principles of $\mathrm{MIH}$ (Table 2). The proportions of students that knew the diagnostic principles of MIH were significantly different between academic levels $\left(\lambda^{2}=64.793, p<0.01 ;\right.$ Table 2$)$. All the aetiologies of $\mathrm{MIH}$ (i.e., genetic factors, pregnant and postnatal factors, drug factors, environment factors) were known by 93 students (93/264, 35\%; Table 2). Most students (156/264,59\%) believed that the incidence of MIH was less than $15 \%$. However, the incidence rate was estimated at $15-30 \%$ by 100 students (100/264, 38\%), 30-60\% by 8 students ( $8 / 264,3 \%)$, but no students estimated an incidence above $60 \%$ (Table 2).

Only 3\% (9/264) of respondents had performed an MIH treatment (Table 2). Among these students, most were more inclined to use a pit and fissure seal, instead of fluoride varnish, for teeth mildly damaged by $\mathrm{MIH}$. However, for teeth affected by $\mathrm{MIH}$ with moderate to severe dentin sensitivity, but no pulp symptoms, no one treatment was favored significantly more than the other choices (Table 2).

Among the respondents that had observed MIH clinically, most (66/104,63\%) were confident in distinguishing $\mathrm{MIH}$ from dental fluorosis, dentin hypoplasia, and other developmental dental diseases (Table 2). The probability of observing MIH clinically was $13 \%(13 / 104)$. The probabilities that students would encounter MIH clinically at frequencies of once per week, once per month, once per 6 months, once per year, and once per $>1$ year were 13\% (13/104), 34\% (35/104), 38\% (39/104), 7\% (7/104), and 10\% (10/104), respectively (Table 2 ). 
The vast majority of students $(296 / 328,90 \%)$ thought it was necessary to add MIH to the curriculum for future systematic teaching (Table 3). They felt that the most desirable parts to learn were the clinical manifestations, differential diagnosis, and treatment methods (Table 2). The most important ways to learn this knowledge were from textbooks $(268 / 328,82 \%)$, the literature $(212 / 328,65 \%)$, the internet $(212 / 328,65 \%)$, and lectures $(169 / 328,52 \%$; Table 2$)$.

\section{Discussion}

At present, the incidence of $\mathrm{MIH}$ in China is quite high ${ }^{5,6}$. There are large differences in incidence rates between cities, possibly due to differences in populations, research methods, diagnostic criteria, etc. Compared to unaffected children, the children with MIH had higher risks of caries and required more dental treatments ${ }^{15}$. Severe $\mathrm{MIH}$ can affect both the quality of life and oral function in children ${ }^{16}$. Without intervention, after two years, molars affected by mild $\mathrm{MIH}$ progress to moderate or severe $\mathrm{MIH}$, with enamel breakdown ${ }^{17}$. If we do not initiate preventive measures, the cost of treating $\mathrm{MIH}$ will become a heavy burden on the country and the individuals ${ }^{18}$. Therefore, it is important to study the etiology, treatment, and awareness of $\mathrm{MIH}$.

Judging from the current data, the situation is not optimistic. Although $80 \%$ of students had heard of $\mathrm{MIH}$, only $20 \%$ of all respondents could accurately evaluate it. Student enthusiasm for learning $\mathrm{MIH}-$ related knowledge was very high, which showed that we need to increase the teaching of MIH-related theoretical knowledge.

For the question of "How often do you encounter MIH clinically?", the results showed a far lower frequency than the frequency experienced by the authors in encountering patients with MIH in clinical settings. This discrepancy might be explained by several reasons. First, the authors have only worked in the pediatric dentistry department; thus, we have come into contact with a large number of patients with $\mathrm{MIH}$. Second, the authors had a better understanding of the theoretical knowledge of $\mathrm{MIH}$. In contrast, the respondents were studying different majors and lacked sufficient opportunities to encounter patients with $\mathrm{MIH}$ clinically or to study MIH systematically.

We found no difference among the different academic levels in wishing to learn more about MIH. Most students desired to learn the clinical manifestations, differential diagnosis, and treatment for $\mathrm{MIH}$. That result reflected the urgent clinical need for knowledge about the principles of $\mathrm{MIH}$ diagnosis and treatment.

The different academic levels acquired knowledge through different sources. As the respondents aged, they gradually changed their ways of acquiring knowledge, from textbooks, to journals, to lectures, and then, to social platforms (e.g., blog). On one hand, this trend reflected the convenience of current networks; on the other hand, it also reflected the dentist's growth process, from passive learning to active learning. By searching for reading materials, students can improve their knowledge reserve and build their own experience system. 
In the survey, nearly $40 \%$ of interviewees had observed MIH clinically, but only $10 \%$ had experienced treating $\mathrm{MIH}$. At present, there is no global consensus on $\mathrm{MIH}$ treatment methods, and treatment principles often lack clear indicators. In 2017, Steffen et al. proposed an MIH treatment-need index (MIH$\mathrm{TNI}$ ) with an $\mathrm{MIH}$ treatment-need coefficient, which could assist in establishing an MIHtreatment system ${ }^{19}$.

\section{Conclusion}

The present study showed that students in the School of Stomatology, Wuhan University had some understanding of $\mathrm{MIH}$, but the knowledge was not comprehensive. They had a weak grasp of key diagnostic and treatment aspects, but there is a strong demand for learning more about $\mathrm{MIH}$. Therefore, based on our research results, we recommend that comprehensive, systematic training on MIH should be required at the undergraduate level, and further training should be offered at the post-graduate level.

\section{Declarations}

\section{Ethics approval and consent to participate}

This study was approved by the Medical Ethics Committee of the School of Stomatology, Wuhan University (\#HGGC-076). Participants were consented to participate in writing, and submitted the completed questionnaire. The research was conducted in full accordance with the World Medical Association Declaration of Helsinki.

\section{Availability of data and materials}

The dataset used and/or analyzed during the current study is available from the corresponding author on reasonable request.

\section{Consent for publication}

The participants were consented in writing to publish the collected data.

\section{Acknowledgements}

We sincerely thank all the dental students for participating in this survey.

\section{Funding}

This work was funded by the National Natural Science Foundation of China (Grant No. 81970904 to M.H.).

\section{Authors' Contributions}


$\mathrm{MH}$ made conception and designed the study. YCL performed the analysis and interpretation of data and drafted the manuscript. $\mathrm{MH}$ edited the manuscript and gave the final approval. All authors reviewed the manuscript.

\section{Competing interests}

The authors declare that they have no competing interests.

\section{References}

1. Weerheijm KL, Jälevik B, Alaluusua S. Molar-Incisor Hypomineralisation. Caries Res. 2001;35:390391.

2. Weerheijm KL, Duggal M, Mejare I, et al. Judgement criteria for molar incisor hypomineralisation (MIH) in epidemiologic studies: a summary of the European meeting on MIH held in Athens, 2003. Eur J Paediatr Dent. 2003;4:110-113.

3. Santos M, Maia LC. Molar Incisor Hypomineralization: Morphological, Aetiological, Epidemiological. In: Contemporary Approach to Dental Caries. InTech; 2012:443-466.

4. Zhao D, Dong B, Yu D, Ren Q, Sun Y. The prevalence of molar incisor hypomineralization: evidence from 70 studies. Int J Paediatr Dent. 2018;28:170-179.

5. Li J, Li L. Investigation of molar-incisor hypomineralization among children from 6 to 11 years in Lucheng District, Wenzhou City. Shanghai J Stomatol. 2012;021:576-579.

6. Sui W, Wu H, Yuan J, Yao H, Hong Y. Current situation and influencing factors of molar-incisor Hypomineralization in primary school students in Soozhow China. Chin Conserv Dent. 2017;027: 103107.

7. Dantas-Neta NB, Moura LF, Cruz PF, et al. Impact of molar-incisor hypomineralization on oral healthrelated quality of life in schoolchildren. Braz Oral Res. 2016;30:e117.

8. Ebel M, Bekes K, Klode C, Hirsch C. The severity and degree of hypomineralisation in teeth and its influence on oral hygiene and caries prevalence in children. Int J Paediatr Dent. 2018;28:648-657.

9. Tagelsir A, Dean JA, Eckert GJ, Martinez-Mier EA. U.S. Pediatric Dentists'Perception of Molar Incisor Hypomineralization. Pediatr Dent. 2018;40:272-278.

10. Gambetta-Tessini K, Mariño R, Ghanim A, Calache H, Manton DJ. Knowledge, experience and perceptions regarding Molar-Incisor Hypomineralisation (MIH) amongst Australian and Chilean public oral health care practitioners. BMC Oral Health. 2016;16: 75.

11. Hussein AS, Ghanim AM, Abu-Hassan MI, Manton DJ. Knowledge, management and perceived barriers to treatment of molar-incisor hypomineralisation in general dental practitioners and dental nurses in Malaysia. Eur Arch Paediatr Dent. 2014;15:301-307.

12. Silva MJ, Alhowaish L, Ghanim A, Manton DJ. Knowledge and attitudes regarding molar incisor hypomineralisation amongst Saudi Arabian dental practitioners and dental students. Eur Arch Paediatr Dent. 2016;17:215-222. 
13. Elhennawy K, Anang M, Splieth $\mathrm{C}$, et al. Knowledge, attitudes, and beliefs regarding molar incisor hypomineralization (MIH) amongst German dental students. Int J Paediatr Dent. 2020.

14. Ghanim A, Silva MJ, Elfrink MEC, et al. Molar incisor hypomineralisation (MIH) training manual for clinical field surveys and practice. Eur Arch Paediatr Dent. 2017;18:225-242.

15. Jalevik B, Klingberg G. Treatment outcomes and dental anxiety in 18-year-olds with MIH, comparisons with healthy controls - a longitudinal study. Int J Paediatr Dent. 2012;22:85-91.

16. Velandia LM, Alvarez LV, Mejia LP, Rodriguez MJ. Oral health-related quality of life in Colombian children with Molar-Incisor Hypomineralization. Acta Odontol Latinoam. 2018;31:38-44.

17. Neves AB, Americano GCA, Soares DV, Soviero VM. Breakdown of demarcated opacities related to molar-incisor hypomineralization: a longitudinal study. Clin Oral Investig. 2019;23:611-615.

18. Schneider PM, Silva M. Endemic Molar Incisor Hypomineralization: a Pandemic Problem That Requires Monitoring by the Entire Health Care Community. Curr Osteoporos Rep. 2018;16:283-288.

19. Steffen R, Kramer N, Bekes K. The Wurzburg MIH concept: the MIH treatment need index (MIH TNI): A new index to assess and plan treatment in patients with molar incisior hypomineralisation (MIH). Eur Arch Paediatr Dent. 2017;18:355-361.

\section{Tables}

Due to technical limitations, Tables 1, 2 and 3 are only available as a download in the Supplemental Files section.

\section{Supplementary Files}

This is a list of supplementary files associated with this preprint. Click to download.

- Tables.docx 